\title{
Design de Visualizações Narrativas Interativas
}

\author{
Caroline Queiroz Santos \\ UFVJM, Departamento de Computação \\ Diamantina, Brasil \\ caroline.queiroz@ufvjm.edu.br
}

\author{
Milene Selbach Silveira \\ PUCRS, Escola Politécnica \\ Porto Alegre, Brasil \\ milene.silveira@pucrs.br
}

\begin{abstract}
Narrative visualization is an emerging data visualization field and has been used for the purpose of communicating information efficiently and intuitively as well as providing greater engagement in data exploration. In this mini-course, we will present and discuss fundamental concepts for interactive narrative visualizations design, and explore these concepts - in theory, and practice - by means of a model that aims to enable reflection on decisions to be taken in narrative visualization's design.
\end{abstract}

\section{ACM Classification Keywords}

H.5.2. Information Interfaces and Presentation: Miscellaneous

\section{Author Keywords}

Visualização de dados; Narrativas; Design

\section{APRESENTAÇÃO}

O objetivo da visualização de dados é auxiliar no processo de compreensão dos dados, aproveitando o sistema visual humano para identificar padrões, tendências e anomalias [7]. Visualizações bem concebidas podem substituir cálculos cognitivos por simples inferências de percepção e melhorar a compreensão, memória e tomadas de decisão [6]. Ao tornar os dados mais acessíveis e atraentes, as visualizações podem envolver diferentes públicos na sua exploração e análise. Desta forma, podemos considerar a visualização como uma espécie de tradutor de dados para uma linguagem de mais fácil compreensão, utilizando gráficos, tabelas, cores, entre outros recursos visuais. O desafio é criar visualizações eficazes e envolventes, apropriadas para o propósito de cada análise a ser realizada [7, 6].

Embora as ferramentas de visualização de dados disponíveis no mercado ofereçam aos seus usuários diversos recursos para a geração de representações gráficas, elas, geralmente, não oferecem alternativas de personalização. Essas ferramentas

Permission to make digital or hard copies of all or part of this work for personal or classroom use is granted without fee provided that copies are not made or distributed for profit or commercial advantage and that copies bear this notice and the full citation on the first page. Copyrights for components of this work owned by others than the author(s) must be honored. Abstracting with credit is permitted. To copy otherwise, or republish, to post on servers or to redistribute to lists, requires prior specific permission and/or a fee. Copyright 2018 SBC.

IHC 2018, Anais Estendidos do XVII Simpósio Brasileiro sobre Fatores Humanos em Sistemas Computacionais.

Outubro 22-26, 2018, Belém, Brasil

Minicursos também não permitem a livre ordenação das visualizações conforme o desejo de seu autor, que, se assim tivesse liberdade, poderia priorizar um ponto de vista, realçar um argumento e apresentar à sua audiência uma nova forma de ver esses mesmos dados, valendo-se de sua habilidade de "contar histórias" por meio de visualizações narrativas.

Há pouco mais de 15 anos, pesquisadores [4, 10, 2, 8, 3, 1] começaram a sugerir que storytelling poderia ser usado para estimular o engajamento e a interação do usuário na exploração de dados. Storytelling refere-se à expressão "contar uma história" e significa, resumidamente, uma sequência ordenada de fatos que pode conter palavras, imagens, visualizações, vídeos ou qualquer uma das suas combinações [8]. Gershon e Page [4] foram os primeiros pesquisadores a perceber que a narrativa poderia contribuir para a visualização da informação e sua principal contribuição foi conceituar a ideia. Em 2010, o tópico apareceu novamente com Segel e Heer [10], que afirmaram que histórias de dados são um tipo emergente de visualizações.

A partir da análise de trabalhos sobre visualização de dados com narrativas e entendimento dos elementos que os compunham, Ghidini [5] elencou sete componentes considerados fundamentais para a construção de visualizações narrativas: os dados, os elementos da narrativa, as informações, os gêneros, os elementos visuais, os elementos estruturais e as técnicas de visualização. $\mathrm{O}$ autor construiu um modelo de representação de narrativas através de visualizações interativas, com dados extraídos de redes sociais. A proposta deste minicurso é refletir sobre o design de visualizações narrativas interativas e, para isso, utilizaremos um modelo, denominado CIViS (Customizable Interface model to support building narrative Visualizations of data extracted from Social media) [9], que sugere caminhos para a reflexão e tomada de decisão em relação aos componentes citados acima, à análise da base de dados, aos modelos de visualização narrativa, à cena e ao sistema de ajuda.

O CIViS é uma estrutura descritiva destinada a apoiar o design de visualizações narrativas, pois proporciona o entendimento sobre o problema em questão (a criação de representações gráficas para um conjunto de dados) e as suas implicações, bem como a reflexão sobre as possíveis soluções a serem criadas. Seu uso é apoiado por um conjunto de cartas representando os componentes, a fim de ajudar os usuários no processo de reflexão e tomada de decisões. 


\section{SUMÁRIO ESTENDIDO}

A proposta deste minicurso consiste em introduzir o conceito de visualização narrativa, discutir e refletir sobre decisões de design para esse tipo de visualização. Para isso, ele será dividido em dois momentos.

\section{Primeiro Momento}

Na primeira parte do minicurso, com duração aproximada de uma hora, serão apresentados e discutidos conceitos fundamentais para o projeto de visualizações narrativas interativas:

- Visualização narrativa. O que é; quando e como surgiu; como está sendo utilizada; como estão as pesquisas nessa área; exemplos reais de visualizações narrativas; tecnologias que podem ser utilizadas para construção deste tipo de visualização.

- Modelo CIViS. O que é; por que vamos utilizá-lo no minicurso; como foi concebido; os componentes; o protótipo; o fluxo de ações.

\section{Segundo Momento}

Na segunda parte, com duração aproximada de duas horas, os participantes serão divididos em pequenos grupos e cada grupo receberá um problema e um conjunto de dados diferentes (advindos de coletas de dados de mídias sociais e de dados governamentais abertos).

Com base nestes dados (e problema), os grupos receberão a tarefa de fazer a modelagem de uma visualização narrativa interativa, e orientações sobre como poderão refletir sobre as histórias que poderiam contar a partir dos referidos dados. Durante a realização da tarefa, eles poderão consultar o tutorial sobre o modelo CIViS e também poderão interagir com as instrutoras do minicurso para esclarecer as dúvidas que tiverem.

Finalizadas as tarefas de cada grupo, os participantes serão convidados a apresentar o resultado de seus trabalhos e a discutir sobre como o modelo pode apoiar o design de visualizações narrativas interativas.

\section{PÚBLICO-ALVO}

Este minicurso visa atender os seguintes perfis de público:

- Alunos (graduação e pós-graduação) e pesquisadores em Interação Humano Computador e Visualização de dados; e

- Pessoas interessadas em conhecer mais sobre visualizações narrativas, como jornalistas, analistas de dados, analistas de mídias sociais, entre outros.

\section{BIOGRAFIA DOS(AS) AUTORES(AS)}

Caroline Queiroz Santos é professora da UFVJM, em Diamantina (MG). Doutora em Ciência da Computação pela PUCRS em 2018. Seus interesses de pesquisa são: Visualização de Dados, Interação Humano-Computador, Narrativas, Computação Social e Engenharia de Software. Lattes: http://lattes . cnpq . br/1055329189606238

Milene S. Silveira é professora associada da PUCRS, em Porto Alegre (RS). Doutora em Ciência da Computação pela PUCRio em 2002. Seu principal interesse de pesquisa é a Interação Humano-Computador, com foco em suas relações com Visualização de Dados, Expressões de Usuários Finais, Redes Sociais e Educação. Lattes: http://lattes . cnpq . br/0483707899231728

\section{DURAÇÃO E IDIOMA}

Este minicurso terá duração de 3 horas e será oferecido em português.

\section{INFRAESTRUTURA E MATERIAIS NECESSÁRIOS}

Sala com carteiras; Lousa e canetas ou giz; Projetor; Internet; Post-its; Flipchart; Canetas do tipo hidrocor (coloridas).

\section{REFERÊNCIAS}

1. Jeremy Boy, Francoise Detienne, and Jean-Daniel Fekete. 2015. Storytelling in Information Visualizations: Does It Engage Users to Explore Data?. In Proceedings of the 33rd Annual ACM Conference on Human Factors in Computing Systems (CHI '15). ACM, New York, NY, USA, 1449-1458.

2. Micheline Elias, Marie-Aude Aufaure, and Anastasia Bezerianos. 2013. Storytelling in Visual Analytics Tools for Business Intelligence. In Human-Computer Interaction - INTERACT 2013, Paula Kotzé, Gary Marsden, Gitte Lindgaard, Janet Wesson, and Marco Winckler (Eds.). Lecture Notes in Computer Science, Vol. 8119. Springer Berlin Heidelberg, 280-297.

3. Ana Figueiras. 2014. How to Tell Stories Using Visualization. In Information Visualisation (IV), 2014 18th International Conference on. 18-18.

4. Nahum Gershon and Ward Page. 2001. What Storytelling Can Do for Information Visualization. Commun. ACM 44, 8 (Aug. 2001), 31-37.

5. Eduardo Ghidini. 2017. Representação de narrativas interativas por meio de visualizações com dados extraídos de redes sociais. Master's thesis. Faculdade de Informática - PUCRS, Porto Alegre, RS, Brasil. Dissertação de Mestrado. Programa de Pós-Graduação em Ciência da Computação.

6. Jeffrey Heer, Michael Bostock, and Vadim Ogievetsky. 2010. A Tour Through the Visualization Zoo. Queue 8, 5, Article 20 (May 2010), 20:20-20:30 pages.

7. Jeffrey Heer, Fernanda B. Viégas, and Martin Wattenberg. 2007. Voyagers and Voyeurs: Supporting Asynchronous Collaborative Information Visualization. In Proceedings of the SIGCHI Conference on Human Factors in Computing Systems (CHI '07). ACM, New York, NY, USA, 1029-1038.

8. Robert Kosara and Jock Mackinlay. 2013. Storytelling: The Next Step for Visualization. Computer 46, 5 (2013), 44-50.

9. Caroline Queiroz Santos. 2018. CIViS: modelo de design de interface customizável para apoiar a construção de visualizações narrativas interativas de dados extraídos de mídias sociais. Ph.D. Dissertation. Escola Politécnica PUCRS, Porto Alegre, RS, Brasil.

10. Edward Segel and Jeffrey Heer. 2010. Narrative Visualization: Telling Stories with Data. Visualization and Computer Graphics, IEEE Transactions on 16, 6 (Nov 2010), 1139-1148. 